\title{
An Architecture for Implementing Application Interoperation with Heterogeneous Systems
}

\author{
George Hatzisymeon ${ }^{1}$, Nikos Houssos ${ }^{2}$, Dimitris Andreadis ${ }^{3}$, \\ and Vasilis Samoladas ${ }^{1}$ \\ ${ }^{1}$ Tech. U. of Crete \\ \{george, vsam\}@softnet.tuc.gr \\ ${ }^{2}$ Communication Networks Laboratory, University of Athens \\ nhoussos@di.uoa.gr \\ ${ }^{3}$ JBoss Europe \\ dimitris@jboss.org
}

\begin{abstract}
We are concerned with the issues faced by software developers with a certain family of distributed applications; those that connect to and interoperate with a heterogeneous infrastructure, i.e., a large heterogeneous collection of external systems (databases, embedded devices, network equipment, internet servers etc.) using different communication protocols. This product family includes applications such as e-commerce systems, network management applications and Grid-based collaborations. For such applications, implementing the interoperation logic is both challenging and expensive. We discuss the major concerns that contribute to the problem, such as transaction support, security and management, as well as integration with workflow or component frameworks. We propose an architecture and related development methodology, based on generative programming, to reduce implementation complexity, allow for rapid application development, ease deployment and manageability.
\end{abstract}

\section{Introduction}

In recent years, there is an increasing tendency for automation of complicated distributed processes whose realisation has previously included a significant degree of human intervention. Of particular interest to us are those activities that involve multiple administrative domains, depend on heterogeneous infrastructures and are subject to frequent change. Relevant examples can be found in diverse fields like Business-to-Consumer (B2C) and Business-to-Business (B2B) e-commerce, service management and provisioning in wired and wireless networks, Grid-based collaborations and computer-aided manufacturing, to name but a few. One of the most challenging aspects of automation is the seamless cooperation of the application logic with a variety of external systems, such as enterprise applications (e.g., ERP, CRM, Billing), databases, Internet/Intranet servers (e.g., web, email, FTP), and embedded devices (network equipment, sensors, instruments etc.) The development of modules 
that interoperate with such systems is tedious and time-consuming, since a lot of effort needs to be put on implementing the required communication/access protocols and data transformations.

The aforementioned developer responsibilities are facilitated by tools that enable the programmer to work at a relatively higher level of abstraction, ranging from simple libraries (e.g., email, FTP or SNMP clients) to powerful middleware (e.g., CORBA, JDBC). However, even with the utilisation of such tools, the task remains challenging, for at least three reasons. First, programmers still need to be aware of a variety of different APIs and technologies, which are irrelevant to the actual task to be implemented. Second, the integration of the external systems into the application frequently requires support for advanced features; dynamic pluggability, transactional execution (when it is possible to undo actions), concurrency control, manageability and configurability. Third, to make external systems available to application logic, they must be made accessible via specialized interfaces: as workflow activities (nodes), web services, component objects, and so forth. This is achieved via suitable wrappers that can be tedious to compose and maintain by hand.

The present contribution aims to provide a framework for realising interaction with heterogeneous infrastructures that minimises the effort required for the development of the interaction logic. In particular, it defines a component architecture and related mechanisms that provide the following capabilities:

- Rapid development of "one-of-a-kind" components to interoperate with external systems, based on generative programming techniques [10] utilizing an active library [25] of access mechanisms (e.g., telnet, FTP, HTTP, JDBC, SNMP).

- On-the-fly deployment and integration of components with the underlying transaction, management and security infrastructures of the application.

- Access to interoperation components from different types of business logic implementations (e.g., workflows, web/grid services) through generatively created wrappers.

To develop our framework, we were guided by the identification of a product family, or domain (in the sense of $[10,11]$ ), namely that of applications which interoperate with a large, or frequently changing, collection of heterogeneous systems. In this domain, development and maintenance costs of interoperation are comparable, or even dominate, development and maintenance costs of application logic. Our contribution includes a refinement of the semantic content of access mechanisms/protocols (domain analysis, in software reuse parlance) and a proposed domain architecture. To validate our approach, an operational prototype has been developed, making use of commercial component frameworks (JBoss [3, 4]) and software engineering tools (Eclipse platform [6], Velocity generator [5]). The prototype has been successfully employed to provide application interoperation with relational databases, network elements and Internet servers.

The rest of the current document is organized as follows: Section 2 presents related work. Section 3 provides an overview of the proposed architecture and elaborates on vital mechanisms such as the task of integrating into an application atomic functional elements and techniques for their template-based, rapid development through 
predefined adaptors. Section 4 discusses the main choices and trade-offs involved in the design of our solution. The last section of the paper is devoted to summary/ conclusions and identification of important elements for further work.

\section{Related Work}

Our work addresses interoperability issues of distributed applications composed by a possibly dynamic, heterogeneous collection of external systems. Such issues have been addressed before in fundamental work in distributed systems, especially in the area of middleware. The bulk of the work can be cast into two broad approaches: (a) general-purpose, low-level mechanisms, such as basic middleware, and (b) application-specific, high-level techniques.

The first approach, which is typified by traditional middleware (RPC, CORBA, RMI, etc.) has been broadly studied. The general direction of the work is to abstract IPC and networking facilities into a high-level application framework. Recent progress in this area has broadened applicability in challenging cases, such as realtime and embedded applications $[13,16]$. Composition of communication protocols has also been studied, notably in the BAST system [15] and in [23, 26]. These techniques are very broadly applicable, but focus on the communication task, and have not been integrated with the higher-level aspects of application frameworks, such as transaction, security and management. The recent introduction of Web Services has advocated a new style of loose integration of autonomous systems, the so-called Service-Oriented Architecture. The platform is currently being augmented with additional conventions related to high-level application aspects (e.g. transactions [8] and resources [12]). It has also been adopted as the standard paradigm for the development of the Grid [13].

The second approach in system interoperability took an application-oriented view of the problem, where the goal was to integrate external systems as close to the application logic as possible. The most notable advances have been in the area of information system integration. The introduction of widely used wrapper technologies (ODBC, JDBC etc.) allowed uniform access to multiple external systems using highlevel languages (such as SQL). This has enabled technologies such as mediator-based information system integration [24] over heterogeneous environments, and objectrelational mapping technologies (e.g. Enterprise JavaBeans).

What is needed today is the convergence of the two approaches outlined above: general-purpose, high-level system interoperation mechanisms. Ambitious software engineering efforts (notably OMG's Model Driven Architecture [20]) are underway to combine current techniques. At the same time, an array of component-based application frameworks are being developed for web (JSP), client-server applications (J2EE), web and grid services (e.g. Globus [13]), mobile agents (e.g. Cougaar [16]), peer-to-peer systems (e.g. PROST [21]), bringing forward new generations of largescale distributed systems. In each of these frameworks there is need for high-level interoperability with external systems, integrated with fundamental transactional, security and management mechanisms. Existing technologies to these directions do exist (e.g. the J2EE Connection Architecture [2]), but they are still little more than hooks into the platform functionality. 


\section{Architecture}

In this section we present our framework in considerable detail. First, we focus on the overall system architecture, introducing fundamental concepts and design. Next, emphasis shifts to application lifetime cycle and the issues thereof.

\subsection{Overall Architecture}

Access to external systems is accomplished through actions, a semantically high-level interface, whose purpose is to isolate application logic from communication and other access concerns as much as possible. Actions have a signature; they accept and return typed arguments, and raise exceptions. Actions must coordinate via concurrency control and transactions. They must implement access control, and perhaps obey other security-related constraints. They must be manageable and discoverable. Finally, they must be accessible in a variety of ways: through workflows, embedded script languages, components (e.g., servlets, EJBs), as exported services, and so forth.

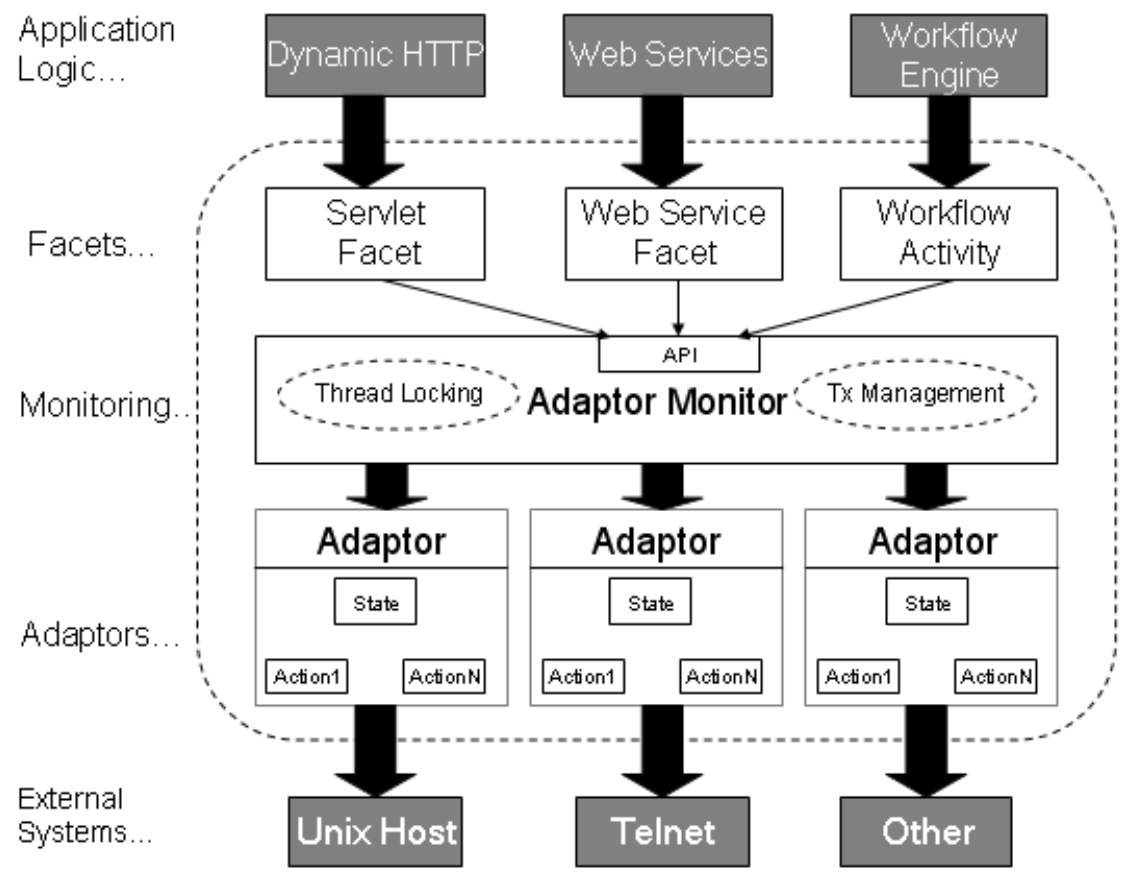

Fig. 1. Making external systems (grey boxes on the bottom) available to application logic (grey boxes on the top): The overall architecture

Based on these concerns we suggest a 3-tier architecture to address the problem. The bottom tier encapsulates external system access specifics: communication channels, protocol implementations, session authorization/login, fault tolerance etc. 
The middle tier's purpose is to integrate with the application framework in use (e.g., J2EE, .NET) and provide synchronized and transactional access to the bottom tier. Finally, the top tier implements different interfaces to the lower tiers (workflows, embedded scripting, web service/CORBA/servlet calls, session EJBs etc.). Our proposed architecture along these lines is depicted in Fig. 1.

Adaptors. The logic for application connectivity to external systems is embedded within adaptors. An adaptor is a component, which encapsulates the necessary connection state and logic to one or more external systems. Adaptors possess two distinct interfaces. The first is a transactional, high-level interface, consisting of actions. This interface is accessed by application logic through the facets (the top layer), and integrates with the underlying application framework, i.e., transaction processing, concurrency control and authorization. The second is a non-transactional, low-level interface, which is only available to the Adaptor Monitor (the middle layer). This interface is used to perform management operations on the adaptor (e.g., resource and connection management and monitoring, security, auditing, on-line configuration and lifecycle management).

Adaptors can relate to external systems or services in a variety of ways. For example, an adaptor may encapsulate a telnet session to a remote Unix host, a TCP/IP multicast group, a Kerberos-authenticated database session, an SNMP-managed device, etc. As a general principle, adaptors are protocol-oriented; they derive from protocol templates, specialized and refined appropriately to comply with application requirements.

Actions. Actions correspond to operations on external systems. Each action is contained within a specific adaptor. Actions are stateless components whose invocations are atomic with respect to application transactions; thus it is desirable that they map to atomic operations on the external system. Each action is specified by a pair of procedures, the first procedure implementing the operation, and the second, which is optional, reversing the operation. These two procedures correspond to the well-known DO-UNDO transactional protocol [16].

In contrast to adaptors, which relate closely to the external system, actions relate to the application logic. Consider for example an adaptor encapsulating a telnet session to a Unix host. The adaptor is responsible for communication-level properties, such as IP address and port, session authentication (login/password exchange), configuration of the conversational exchange (e.g., recognising the session's command prompt), etc. Actions related to this particular adaptor are totally application-specific. For example, if the purpose of connecting to this Unix host is to perform user management on it, sample actions for this adaptor would include adduser, deluser, chgpass, and chgshell. The developer would be responsible for implementing these actions (and their reversals) as required by the host, e.g., compose the command line necessary to add/delete a user, and parse the command output. The adaptor will only provide a protocol-specific API (e.g., in our example, an execute function, accepting a command line and returning a stream of the command output).

Adaptor Monitor. Actions are invoked only via the Adaptor Monitor. This module constitutes the middle layer of our architecture and is responsible for application-wide adaptor integration. Primarily, it is a Transaction Processing monitor [16] for action invocations (hence its name). It logs the information needed to reverse the sequence 
of actions of an aborted transaction. This mechanism integrates closely to the application framework.

Concurrency control for action invocations is supported in cooperation with the TP monitor, by two locking mechanisms: (a) synchronization locking, ensuring mutual exclusion among concurrent action invocations from multiple threads, and (b) Consistency locking, where transactions can explicitly obtain long-term locks on specific actions, that will preclude other transactions from invoking them until the locks are released. This mechanism can be used to implement transaction scheduling policies, such as serialization [16].

The Adaptor Monitor offers directory services over the deployed adaptors and actions. Apart from name-based discovery it also provides metadata services for adaptors and actions, both in human-readable form (e.g., to be used by interactive management tools), and API-based (i.e., reflection descriptors of adaptor and action interfaces). Another responsibility of the Adaptor Monitor is adaptor lifecycle management: The adaptor interface includes four mandatory operations: init, start, stop and destroy. Typically, these are automatically invoked upon particular management operations (e.g., adaptor (re-)deployment, system exit/start). During lifecycle operations, the Adaptor Monitor takes into account global sequencing constraints for setting up adaptors. The relevant information is provided at adaptor design time.

Facets. Facets are responsible for application-wide action integration. The Adaptor Monitor has a standard interface for all adaptor and action-related operations, which may not be convenient to call directly from application logic. Some useful types of facets include:

- Workflow facets. Make actions available as activities (workflow nodes) to a workflow engine executing in the application.

- Services facets. Actions/sets of actions become available as Web Services, CORBA or RMI objects etc. to the application and its clients.

- Script facets. Actions become available to application-embedded script languages (e.g., Visual Basic, Python).

- Unit testing facets. Interfacing to the testing and debugging tools.

- Servlets, Java Beans, and other types of application logic components.

Facets are generated automatically from adaptor specifications, using specialized tools for each facet type.

\subsection{Implementing Adaptors}

Adaptors can be very complex components. Their implementation is in most cases based on the knowledge of a specific protocol/domain/language. To avoid development of every new adaptor from scratch, we employ a generative approach that allows for rapid, simplified implementation. Our approach is based on the development of an active library [25] of protocols, i.e., a collection of protocol implementation templates, which encapsulate most of the required connection knowledge, and can be customized and refined through a graphical tool. 


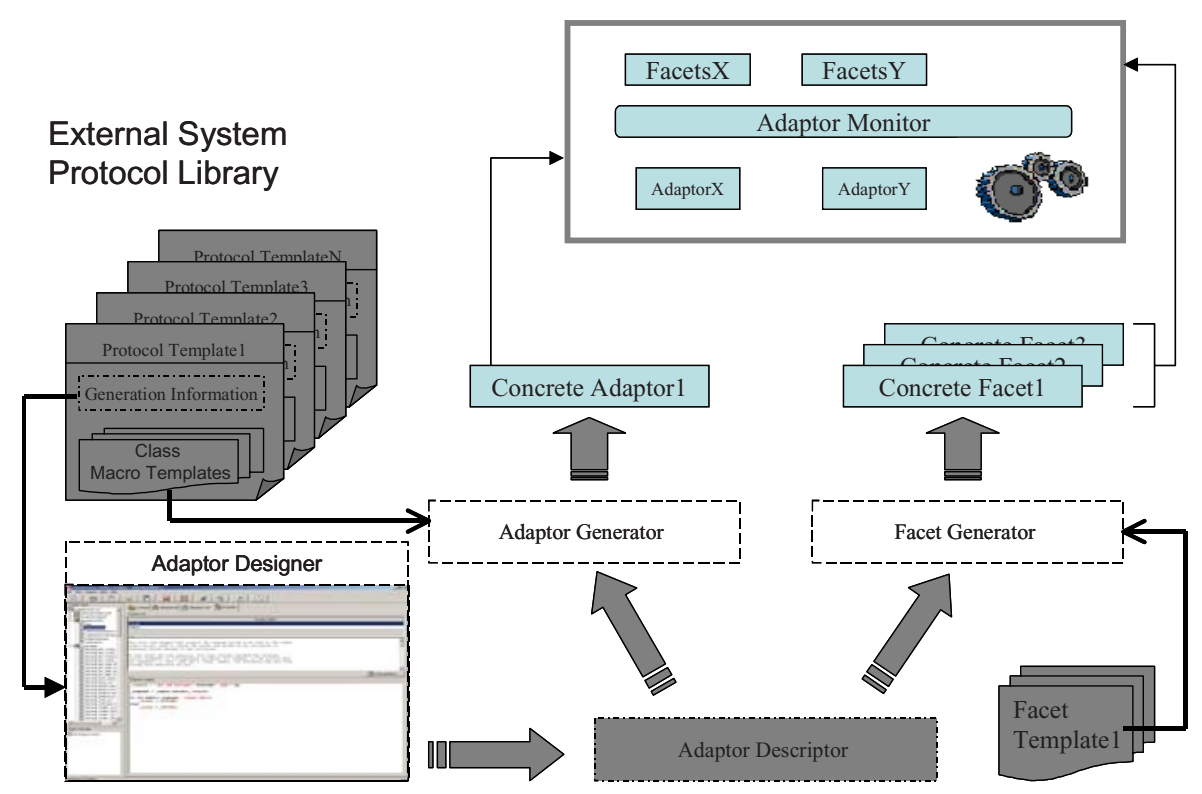

Fig. 2. Adaptor and facet development process

Our adaptor development process is depicted in Fig. 2. The first stage is adaptor design, and is performed graphically using the Adaptor Designer. It comprises of three steps: (a) selection of a protocol template, (b) customization of connection, deployment, lifecycle, authentication and auditing aspects, and (c) implementation of the actions required by the application, which includes, for each action, definition of its signature, implementation of the DO-UNDO logic, locking specification and documentation. The result of this process is an (XML-encoded) Adaptor Descriptor, which is used to drive code generation.

Each protocol template comprises a number of class macro-templates, which contain templatised source code to be fed into the generator, as well as the Generator Information (GI), an XML descriptor of the protocol template. The GI is used to customize the Adaptor Designer to the specific needs of the protocol at hand. It contains a variety of information:

- Protocol information, such as name, API exposed by the protocol implementation and deployment information (e.g., dependencies on external software libraries)

- Adaptor properties: typed attributes exposed to the adaptor API. These can be the mandatory attributes that maintain the state of the adaptor or additional information required to configure protocol-related operation (connection, resource and lifecycle management, authentication etc.)

- Action types: To simplify implementation of actions, each adaptor can support a number of action types. Each action type is specified by a name, a collection of class macro-templates and human-readable documentation of the contract to be supported by action implementations. The contract of an action type consists of 
mandatory in-out arguments and guidelines that act as a reference for coding an action's DO-UNDO procedures, and per action action-type. Action types can provide utilities assisting the most common types of interaction processing (text/XML/URL parsers, data transformers, macro expanders, etc.)

The output of Adaptor Designer is an Adaptor Descriptor (AD), an XML document holding the specification of a concrete adaptor. It provides code generators with a variety of information that they utilise to parameterise the instantiation of code macro-templates. An AD contains data for both the protocol (mostly copied from the GI) and the adaptor. The main part of adaptor information is a list of adaptor properties. These correspond to the attributes defined in the GI, with concrete values provided by the developer. Optionally, additional properties can be specified during adaptor design. Properties can be used on action method implementations, or can be part of the non-transactional adaptor interface.

Important elements of the $\mathrm{AD}$ are the action specifications. Each action specification contains action type, name, action signature, a human-readable documentation of the action interface and semantics, implementations for the DOUNDO procedures, specification of locking behavior, and deployment information (e.g., dependency on external libraries). An AD also encapsulates additional XML metadata that is associated with the adaptor and individual actions, whose semantics are opaque to the framework. This metadata can be accessed both during facet generation, and at runtime through the Adaptor Monitor.

The last step in adaptor implementation is automated by a code generation tool, which receives the Adaptor Descriptor, and uses the code macro-templates of the protocol template to produce source code, deployment metadata, scripts etc. Facets are also generatively produced by the facet generator, based on the adaptor descriptor and a list of appropriate class macro-templates, drawn from the active library.

\subsection{Prototype Implementation}

For an initial implementation of our platform we chose the Java 2 Enterprise Edition platform (J2EE) and the JBoss application server. JBoss provides robust pluggable implementations of Java Management eXtensions (JMX) [1] and the Java Connector Architecture (JCA) [2]. Adaptors are implemented as standard JMX MBeans, providing an interface accessible through JMX.

We have used the Velocity generator [5] to implement both the Adaptor Generator and the Facet Generator. Velocity provides an intuitive macro language that adds only marginal complexity to the coding of protocol templates. The Adaptor Designer is currently a stand-alone Java application, although we plan to implement a new version inside the Eclipse IDE.

We have implemented a moderate library of protocols, including most Internet services (telnet, FTP, http, SMTP, SNMP), as well as three facet types: an Enterprise Java Bean (EJB) facet, where actions are available to EJBs as methods, a Web Service facet, where actions are exposed as Web Services by JBoss, and a jBPM workflow facet, where actions are available as workflow activities. 


\section{Discussion}

The present section provides a discussion on the architecture proposed in this paper. First, we consider its applicability in two different domains: telecommunications service provisioning and grid-based applications. Then, we elaborate on important choices and trade-offs that we faced in the course of the system design.

\subsection{Application Areas}

Service Provisioning. The proposed architecture is particularly suited for service provisioning applications, in the general area of telecommunications Operation Support Systems (OSS). The goal of service provisioning is to automate the provisioning of telecommunication services across different technology domains (traditional land line phone service, internet access, mobile access, etc.) [9]. Some of the major challenges of service provisioning addressed the proposed architecture are as follows:

- Heterogeneity: Providers offer services over a variety of telecommunication equipment and technologies. A typical provisioning scenario may involve interaction with a dozen different devices or management systems.

- Consistency: Activation failures are common in complex systems and they can easily result to wasting valuable network resources if a multi-step activation scenario fails at some intermediate point. Transactional interactions with network devices eliminate the error-prone practice of coding rollback logic by hand.

- Constant change. Every so often, the marketing department will come up with yet another bundle of services sold as a package, at which point the activation flow will need to be adapted or extended. Our architecture matches those requirements because it allows rapid, easy introduction of new actions, or alteration of old ones.

Grid Computing. Grids [13] constitute virtual computation platforms, promising to make available unparalleled levels of computing, storage and communication resources to scientific, engineering and business applications. To fulfil this promise, Grid technology must be able to harness the resources contributed by the participants of a virtual organization. These resources form a heterogeneous infrastructure, the Grid fabric, which must be made accessible to Grid development and application frameworks through a uniform interface, the Grid middleware. Grid-related research has been concerned with the grid middleware and higher-level components: resource management, brokering, semantic discovery, etc. There is relatively little work on integrating fabric resources to grid middleware. In real Grids this is done in ad-hoc ways, with significant cost. Our proposed architecture can reduce this cost, by exposing the Grid fabric to the Grid middleware through adaptors. Thus we can benefit in several ways. Access to resources, applications and datasets, can automatically integrate with transactional, concurrency, semantic/metadata and security mechanisms of the Grid middleware. Semantic issues are of particular interest; brokering and planning performed by Grid middleware depends on a semantic representation of the grid fabric stored in metadata repositories. Suitable facets can be used to easily populate these repositories with minimum effort. 


\subsection{Design Choices and Trade-Offs}

A principal goal of our solution is achieving separation of concerns with regard to development of interoperation logic. This is accomplished through the complementary contribution of three types of actors:

- Framework developer: implements functionality common to all adaptors as well as the development tools (e.g., generators, facet templates), as outlined in this paper. Once the framework is available there is little need for subsequent modifications.

- Connectivity experts: develop specific protocol templates. The implementation of these templates is tedious and requires extensive knowledge of communication and access protocols (e.g., SMTP, FTP, TELNET, JDBC). It is expected that new protocol templates are continuously needed, albeit with moderate frequency.

- Application domain experts: responsible for the application-specific intelligence, i.e., instantiation of adaptors and implementation of actions. This task is normally the easiest and less costly in terms of effort and time. Actions are constantly updated/added to the system, possibly at a high frequency.

The above distinction of roles enables new pieces of connectivity logic to be easily added to an application, so that interoperation requirements are rapidly satisfied.

An important design choice is the dual interface exported by the adaptors, as elaborated in section 3.1. Actions comprise the high-level portion of the adaptor interface, supporting features like transactions, concurrency and authorisation. The rest of the interface is too low-level for the application logic to be aware of; it is available only to the Adaptor Monitor and pertains to management functions. Features like transactionality and concurrency are not supported for these operations; this would considerably complicate matters without any significant benefit. There is ample precedent justifying our choice, e.g., in database systems, where the Data Manipulation Language is transactional, while the Data Definition Language is not.

The ultimate objective of the framework is to enable application logic to invoke actions. An action encompasses only the logic that needs to be executed at the external resource; it does not care how the connectivity is obtained. Furthermore, actions are atomic; they do not encapsulate any further nested actions that can be handled as distinct functions from a transactional point of view. Thus, they need not maintain any state information. Support for transactional behavior is optional. Actions are therefore extremely lightweight components; the simpler among them may consist of only a few lines of code. This leads to minimal effort and time required from the part of the action developer, as well as minimal overhead for the execution of actions. In case of non-reversible actions, the overhead is even smaller, since no invocation history need be preserved.

In designing the transaction support for the Adaptor Monitor, we chose to select DO-UNDO semantics, instead of the more powerful DO-UNDO-REDO semantics. Thus, it will be difficult to implement advanced buffering/caching/coalescing behaviour at the action level. This choice limits performance in a few cases; for example, an object-relational mapping of an external database may be less efficient. On the other hand, we gain in simplicity: for most external systems, the meaning of 
REDO is not obvious. A related concern concerns our choice of locking semantics. We chose not to constrain the user to a specific protocol (an obvious choice would have been two-phase locking) but instead allow application logic to control locking explicitly. If more constrained behaviour is desirable for some adaptors, it can in principle be supported by special facets.

A concern we faced during the design of the overall architecture is the management of events that originate from the underlying infrastructure and are of interest to the application. Relevant issues have been the subjects of extensive research efforts in areas related to distributed systems [27, 28]. The approach adopted by our framework so far does not include an explicit mechanism for event propagation towards the application. However, this can be achieved through polling at the application logic level.

\section{Summary - Future Work}

In this paper, we presented an architecture for application interoperation with heterogeneous infrastructures. Our contributions pertain to applications which have extensive and frequently changing requirements for connection to external resources. Our architecture promotes separation of concerns in the development of interconnection functionality, with a bias in the direction of reducing the burden on the developer of application logic.

With regard to future work, our top priorities include: (a) incorporating event management into the framework, (b) utilisation of the framework in Grid applications based on the Globus platform, and (c) investigation of the architecture implementation on platforms other than J2EE, such as the Cougaar agent framework.

\section{References}

1. Java Management Extensions White Paper: Dynamic Management for the Service Age. http://java.sun.com/products/JavaManagement, 1999.

2. J2EE Connector Architecture Specification, Version 1.5, Nov. 2003.

3. JBoss Open Source Application Server, http://www.jboss.org.

4. M Fleury, F Reverbel, The JBoss Extensible Server, International Middleware Conference (Middleware 2003), Brazil, June 2003.

5. Velocity Template Engine, http://jakarta.apache.org/velocity.

6. Eclipse Integrated Development Environment, http:// www.eclipse.org.

7. A. Beugnard, Communication Services as Components for Telecommunication Applications, In Proc. Objects and Patterns in Telecom Workshop (in ECOOP'00), 2000.

8. L. F. Cabrera, G. Copeland, M. Fwingold et al. Web Services Atomic Transaction (WSAtomicTransaction), Nov 2004.

9. A. Clemm, F. Shen and V. Lee, Generic Provisioning of Heterogeneous Services-a Close Encounter with Service Profiles. Computer Networks 43 (2003), 43-57.

10. K. Czarnecki and U. W. Eisenecker, Components and Generative Programming. In Proc. 7th European Software Eng. Conf., 1998. 
11. A. Egyed, N. Mehta and N. Medvidovic, Software Connectors and Refinement in Family Architectures. In Proc. 3rd Int'1 W. on Development and Evolution of Software Architectures for Product Families, LNCS 1951, 96-105, 2000.

12. I. Foster, J. Frey, S. Graham et al. Modelling Stateful Resources with Web Services. Preliminary whitepaper version $1.1,3 / 5 / 2004$.

13. I. Foster, C. Kesselman, J. Nick, S. Tuecke. Grid Services for Distributed System Integration. Computer, 35(6), 2002.

14. A. Gokhale and D. C. Schmidt, Techniques for Optimizing CORBA Middleware for Distributed Embedded Systems. In Proc. of INFOCOM '99, 1999.

15. B. Garbinato and R. Guerraoui, Flexible Protocol Composition in Bast, In Proc. Int'l Conf. on Distributed Computing Systems (ICDCS), 1998.

16. J. Gray and A. Reuter, Transaction Processing: Concepts and Techniques. 1993, Morgan Kaufmann Pub.

17. A. Helsinger, A. Thome and T. Wright. Cougaar: A Scalable, Distributed Muti-Agent Architecture. In Proc. IEEE Conf. on Systems, Man and Cybernetics (SMC), 2004.

18. A. Krishna, D. C. Schmidt and R. Klefstad, Enhancing Real-Time CORBA via Real-Time Java. In Proc. 24th IEEE Int'l Conf. on Distributed Computing Systems (ICDCS), 2004.

19. N. Mehta, N. Medvidovic and S. Phadke, Towards a Taxonomy of Software Connectors. In Proc. Int'l Conf. on Software Engineering, 178-187, 2000.

20. J. Miller and J. Mukerji (Eds.), Model Driven Architecture (MDA). http://www.omg.org/cgi-bin/doc?ormsc/2001-07-01, 2001.

21. M. Portmann, S. Ardon, P. Senac, A. Seneviratne, PROST: A Programmable Structured Peer-to-peer Overlay Network, In Proc. IEEE Int'l Conf. on Peer-to-peer Computing (P2P), 2004.

22. Y. Smaragdakis and D. Batory, Mixin Layers: An Object-Oriented Implementation Technique for Refinements and Collaboration-Based Designs. Software Engineering and Methodology 11(2), 215-255, 2002.

23. B. Spitznagel and D. Garlan, A Compositional Approach for Constructing Connectors. In Proc. Working IEEE/IFIP Conf. on Software Architecture (WISCA), 2001.

24. S. Thakkar, C. A. Knoblock and J. L. Ambite, A View Integration Approach to Dynamic Composition of Web Services. In Proc. ICAPS Workshop on Planning for Web Services. 2003.

25. T. L. Veldhuizen and D. Gannon. Active libraries: Rethinking the roles of compilers and libraries. In Proc. SIAM Workshop on Object Oriented Methods for Inter-operable Scientific and Engineering Computing (OO'98), 1998.

26. D. M. Yellin and R. E. Strom, Interfaces, Protocols and the Semi-Automatic Construction of Software Adaptors. In Proc. Object-Oriented Programming, Systems, Languages and Architectures (OOPSLA), 176-190, 1994.

27. R. Meier, Taxonomy of Distributed Event-Based Programming Systems, 1st Int'l Workshop on Event-Based Systems (DEBS 2002), July 2002, Vienna, Austria.

28. P. Th. Eugster et al., The Many Faces of Publish/Subscribe, ACM Computing Surveys, Vol. 35, Issue 2, June 2003. 